\title{
Direct Vasocontractile Activities of Bupivacaine Enantiomers on the Isolated Rat Thoracic Aorta
}

\author{
Mai Mukozawa, Ko Takakura, and Maki Mizogami \\ Department of Anesthesiology, Asahi University, 1851 Hozumi, Gifu 5010296, Japan \\ Correspondence should be addressed to Ko Takakura, takakura@dent.asahi-u.ac.jp \\ Received 9 August 2010; Revised 23 September 2010; Accepted 6 October 2010 \\ Academic Editor: Uwe Rudolph
}

Copyright () 2010 Mai Mukozawa et al. This is an open access article distributed under the Creative Commons Attribution License, which permits unrestricted use, distribution, and reproduction in any medium, provided the original work is properly cited.

Background. In vitro studies with isolated arteries have shown direct vasoactivity of racemic bupivacaine. However, there is little information on the direct vasoactivities of bupivacaine enantiomers, $\mathrm{S}(-)$ - and $\mathrm{R}(+)$-bupivacaine. Methods. We performed functional examinations using isolated intact thoracic aortic rings from male Wistar rats. Changes in ring tension produced by $\mathrm{S}(-)-, \mathrm{R}(+)$-, or racemic bupivacaine were measured in Krebs solution. Results. $\mathrm{S}(-)$-bupivacaine produced the strongest contraction of the three agents. $\mathrm{R}(+)$-bupivacaine showed limited vasoconstriction. The effects of racemic bupivacaine were located between these two. Conclusion. Each bupivacaine enantiomer showed specific vasocontractile activity, which affects the activity of racemic bupivacaine.

\section{Introduction}

The local anesthetic bupivacaine is a racemic mixture of $\mathrm{S}(-)$ - and $\mathrm{R}(+)$-enantiomers. Racemic bupivacaine has biphasic vasoactivities, namely, vasoconstriction at a low concentration and vasodilatation at a high concentration [1]. Since 1976, when Aps and Reynolds showed this vasoactivity in a double-blind trial with forearm skin color changes of 31 volunteers [1], these vasoactivities have been further demonstrated using various in vivo methods with various animals or humans, such as television microscopy in rat cremaster muscle microvasculatures [2], intravital microscopy through a spinal window in dog pial vasculatures [3], laser Doppler imaging in human skin [4], as well as other techniques. Although inhibition of sympathetic nerves innervating arteries by racemic bupivacaine could not be ignored in in vivo studies, some in vitro studies with isolated preparations from human umbilical arteries [57], rat uterine arteries [8], and human uterine arteries [9] have confirmed that the vasoactivities are produced by direct actions of racemic bupivacaine itself on the arteries.

$\mathrm{S}(-)$-bupivacaine was developed as an alternative longacting local anesthetic with a clinical profile similar to that of racemic bupivacaine but with a lower potential for producing systemic toxicity [10]. S(-)-bupivacaine also has biphasic vasoactivities similar to those of racemic bupivacaine, which has been shown in in vivo studies [4, 11-13]. Furthermore, some of the in vivo studies have shown that $\mathrm{R}(+)$-bupivacaine produced a dose-dependent vasodilatation $[11,13]$. However, there is little information on the direct vasoactivities of $\mathrm{S}(-)$ - and $\mathrm{R}(+)$-bupivacaine based on in vitro study. In this study, we investigated the vasocontractile activities of these agents using isolated rat aorta.

\section{Methods}

2.1. Animals. The experimental protocol was approved by the institutional animal care committee of Asahi University. Male Wistar rats weighing 240-280 g were used.

2.2. Functional Experiments. Rats were killed by decapitation under sevoflurane anesthesia, and the thoracic aorta was isolated and removed [14]. The thoracic aorta was placed in Krebs-Henseleit solution (mM; $\mathrm{NaCl} 118, \mathrm{KCl} 4.7, \mathrm{NaHCO}_{3}$ $25, \mathrm{KH}_{2} \mathrm{PO}_{4}$ 1.2, $\mathrm{MgSO}_{4}$ 1.2, $\mathrm{CaCl}_{2}$ 2.5, and glucose $10 ; \mathrm{pH}$ 7.4). Aortic rings were carefully prepared under a dissecting microscope, and then each intact ring was carefully pulled by wires in an organ chamber containing $5 \mathrm{~mL}$ Krebs-Henseleit 


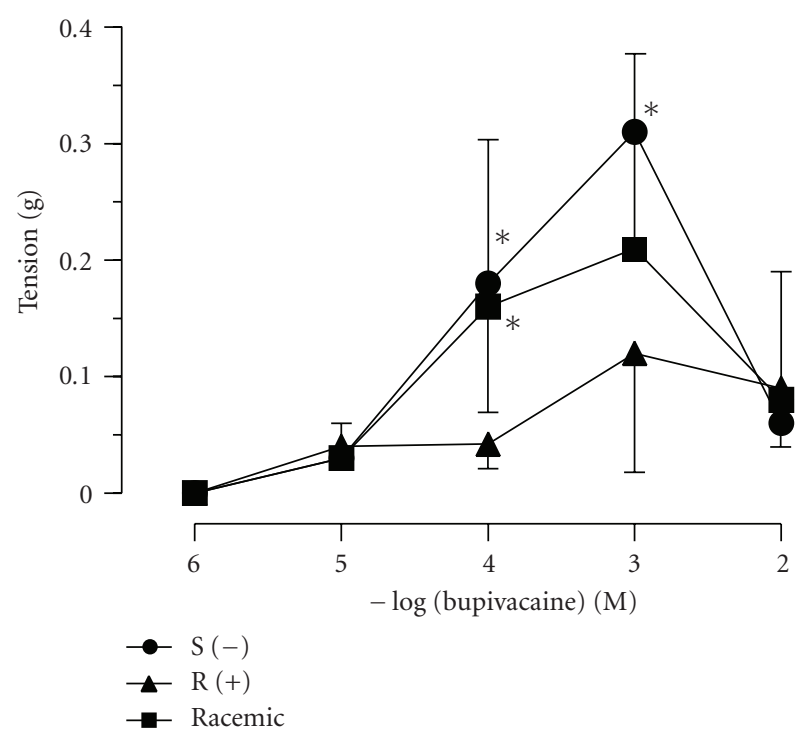

Figure 1: Changes in vascular tension provoked by $\mathrm{S}(-)-, \mathrm{R}(+)-$, and racemic bupivacaines. ${ }^{*}: P<.05$ versus $\mathrm{R}(+)$-bupivacaine. $n=8$.

solution bubbled with $95 \% \mathrm{O}_{2}$ and $5 \% \mathrm{CO}_{2}$ at $37^{\circ} \mathrm{C}$. After a resting tension of $1.0 \mathrm{~g}$ was applied during one-hour equilibration period, changes in the tension were recorded isometrically when $\mathrm{S}(-)$-bupivacaine, $\mathrm{R}(+)$-bupivacaine, or racemic bupivacaine was cumulatively applied. Contractions were expressed as mg contractile tension.

2.3. Chemicals. $\mathrm{S}(-)$-bupivacaine and $\mathrm{R}(+)$-bupivacaine were generously donated by Maruishi Pharmaceutical (Osaka, Japan), and pseudoracemic bupivacaine was prepared by mixing with $\mathrm{S}(-)$-bupivacaine and $\mathrm{R}(+)$ bupivacaine at a ratio of $1: 1[15]$.

2.4. Statistical Analysis. The results are expressed as mean \pm $\mathrm{SD}$. The maximum response $\left(\mathrm{E}_{\max }\right)$ and the concentration producing a half-maximal response $\left(\mathrm{EC}_{50}\right)$ were determined by Finley's probit analysis. Significance of differences was analyzed by Kruskal-Wallis test and Scheffé method as a post hoc comparison for multiple comparisons at a significance level of 0.05 .

\section{Results}

$\mathrm{S}(-)$-bupivacaine, $\mathrm{R}(+)$-bupivacaine, and racemic bupivacaine produced a biphasic response in the aortic rings, namely, concentration-dependent contraction from $10 \mu \mathrm{M}$ to $1 \mathrm{mM}$ and relaxation at higher concentrations $(n=8$, Figure 1.) The vasocontractile responses produced by $\mathrm{S}(-)$ bupivacaine and racemic bupivacaine were significantly stronger than those by $\mathrm{R}(+)$-bupivacaine. Furthermore, there were significant differences among $\mathrm{E}_{\max }$ or $\mathrm{EC}_{50}$ of each bupivacaine (Table 1 ).
TABLE 1: $\mathrm{EC}_{50}$ values and maximum contraction by bupivacaines.

\begin{tabular}{lll}
\hline Bupivacaine & $\mathrm{EC}_{50}\left(\times 10^{-5}\right)$ & $\mathrm{E}_{\max }(\mathrm{g})$ \\
\hline $\mathrm{S}(-)$ & $7.3 \pm 2.9$ & $0.31 \pm 0.06$ \\
$\mathrm{R}(+)$ & $4.1 \pm 4.0^{*}$ & $0.12 \pm 0.10^{*}$ \\
Racemic & $4.1 \pm 1.6^{*}$ & $0.21 \pm 0.10$ \\
\hline
\end{tabular}

$* P<.05$ versus $S(-) . n=8$.

\section{Discussion}

As there has been only limited study of the direct vasoactivities of two bupivacaine enantiomers, $\mathrm{S}(-)$ - and $\mathrm{R}(+)$ bupivacaine, we compared the vasocontractile effects of these two agents with that of racemic bupivacaine in this study. $\mathrm{S}(-)$-bupivacaine showed the strongest $\mathrm{E}_{\max }$ of the three agents, while $\mathrm{R}(+)$-bupivacaine showed limited vasoconstriction. Although racemic bupivacaine produced as much $\mathrm{E}_{\max }$ as $\mathrm{S}(-)$-bupivacaine statistically (Table 1), the activity level of racemic bupivacaine was located between those of $\mathrm{S}(-)$-bupivacaine and $\mathrm{R}(+)$-bupivacaine graphically (Figure 1 ). R(+)-bupivacaine, which produced small vasoconstriction even at high concentration, may interfere with vasoconstriction by $\mathrm{S}(-)$-bupivacaine in racemic bupivacaine, which consists of the two enantiomers. Several in vitro studies have been done on racemic bupivacaine's direct vasoactivities. It has been reported that racemic bupivacaine contracted isolated rat uterine arteries [8], human uterine arteries [9], human umbilical arteries [5-7], and veins [6] in various degrees. Different vasocontractile activities of the enantiomers among the vessels might be, at least, a cause of the variety.

$\mathrm{S}(-)$-bupivacaine produced the strongest vasoconstriction of the three bupivacaines in our study. Considering that the clinical use of $\mathrm{S}(-)$-bupivacaine is increasing because of its lower toxicity [10], it is important to note that it may produce greater vasoconstriction than racemic bupivacaine does. Bupivacaine administered for epidural anesthesia raises the intrathecal and plasma concentration [16] and might contract several important vessels, including the pial, epidural, uterine, umbilical arteries, as well as others, with subsequent decrease of blood flow.

\section{References}

[1] C. Aps and F. Reynolds, "The effect of concentration on vasoactivity of bupivacaine and lignocaine," British Journal of Anaesthesia, vol. 48, no. 12, pp. 1171-1174, 1976.

[2] R. A. Johns, W. C. Seyde, C. A. DiFazio, and D. E. Longnecker, "Dose-dependent effects of bupivacaine on rat muscle arterioles," Anesthesiology, vol. 65, no. 2, pp. 186-191, 1986.

[3] H. Iida, Y. Watanabe, S. Dohi, and T. Ishiyama, "Direct effects of ropivacaine and bupivacaine on spinal pial vessels in canine: assessment with closed spinal window technique," Anesthesiology, vol. 87, no. 1, pp. 75-81, 1997.

[4] D. J. Newton, D. Burke, F. Khan et al., "Skin blood flow changes in response to intradermal injection of bupivacaine and levobupivacaine, assessed by laser doppler imaging," Regional Anesthesia and Pain Medicine, vol. 25, no. 6, pp. 626$631,2000$. 
[5] T. Tuvemo and G. Willdeck-Lund, "Smooth muscle effects of lidocaine, prilocaine, bupivacaine and etidocaine on the human umbilical artery," Acta Anaesthesiologica Scandinavica, vol. 26, no. 2, pp. 104-107, 1982.

[6] E. Monuszko, S. Halevy, K. Freese, M. Liu-Barnett, and B. Altura, "Vasoactive actions of local anaesthetics on human isolated umbilical veins and arteries," British Journal of Pharmacology, vol. 97, no. 2, pp. 319-328, 1989.

[7] H. Bariskaner, S. Tuncer, A. Taner, and N. Dogan, "Effects of bupivacaine and ropivacaine on the isolated human umbilical artery," International Journal of Obstetric Anesthesia, vol. 12, no. 4, pp. 261-265, 2003.

[8] H. Norén, B. Lindblom, and B. Källfelt, "Effects of bupivacaine and calcium antagonists on the rat uterine artery," Acta Anaesthesiologica Scandinavica, vol. 35, no. 1, pp. 77-80, 1991.

[9] H. Norén, B. Lindblom, and B. Källfelt, "Effects of bupivacaine and calcium antagonists on human uterine arteries in pregnant and non-pregnant women," Acta Anaesthesiologica Scandinavica, vol. 35, no. 6, pp. 488-491, 1991.

[10] C. Nau and G. R. Strichartz, "Drug chirality in anesthesia," Anesthesiology, vol. 97, no. 2, pp. 497-502, 2002.

[11] C. Aps and F. Reynolds, "An intradermal study of the local anaesthetic and vascular effects of the isomers of bupivacaine," British Journal of Clinical Pharmacology, vol. 6, no. 1, pp. 6368, 1978.

[12] H. Iida, H. Ohata, M. Iida, K. Nagase, M. Uchida, and S. Dohi, "The differential effects of stereoisomers of ropivacaine and bupivacaine on cerebral pial arterioles in dogs," Anesthesia and Analgesia, vol. 93, no. 6, pp. 1552-1556, 2001.

[13] D. J. Newton, G. A. McLeod, F. Khan, and J. J. F. Belch, "Vasoactive characteristics of bupivacaine and levobupivacaine with and without adjuvant epinephrine in peripheral human skin," British Journal of Anaesthesia, vol. 94, no. 5, pp. 662-667, 2005.

[14] K. Takakura, W. Xiaohong, K. Takeuchi, and S. Fukuda, "Peroxynitrite decreases dopamine's vasoconstrictive activity," Anesthesia and Analgesia, vol. 97, no. 5, pp. 1492-1496, 2003.

[15] M. Mizogami, H. Tsuchiya, T. Ueno, M. Kashimata, and K. Takakura, "Stereospecific interaction of bupivacaine enantiomers with lipid membranes," Regional Anesthesia and Pain Medicine, vol. 33, no. 4, pp. 304-311, 2008.

[16] M. Ratajczak-Enselme, J.-P. Estebe, F.-X. Rose et al., "Effect of epinephrine on epidural, intrathecal, and plasma pharmacokinetics of ropivacaine and bupivacaine in sheep," British Journal of Anaesthesia, vol. 99, no. 6, pp. 881-890, 2007. 


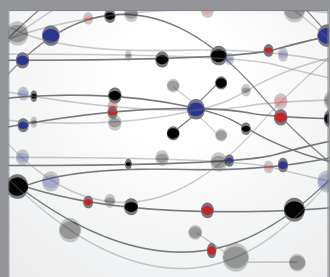

The Scientific World Journal
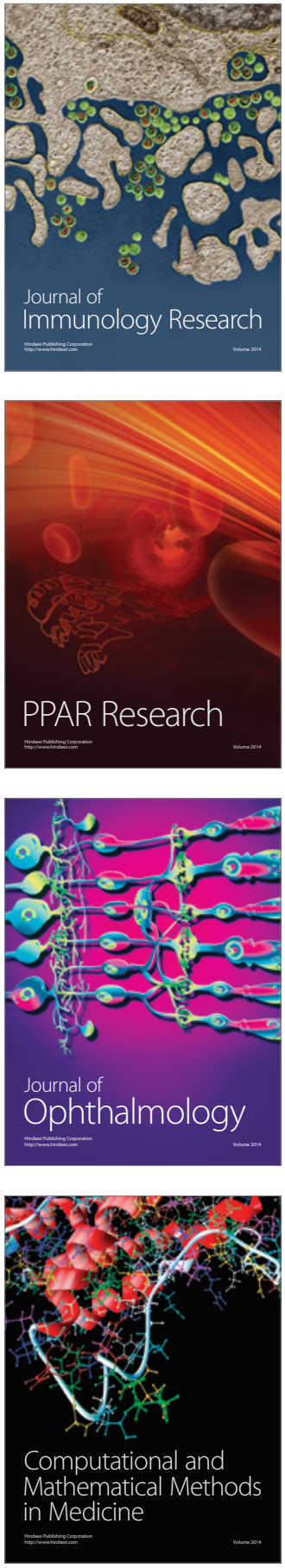

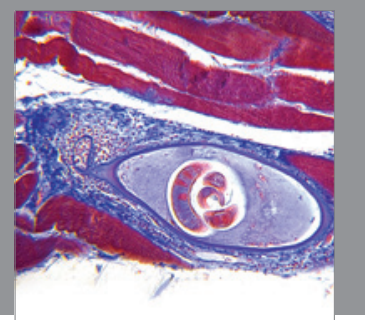

Gastroenterology

Research and Practice
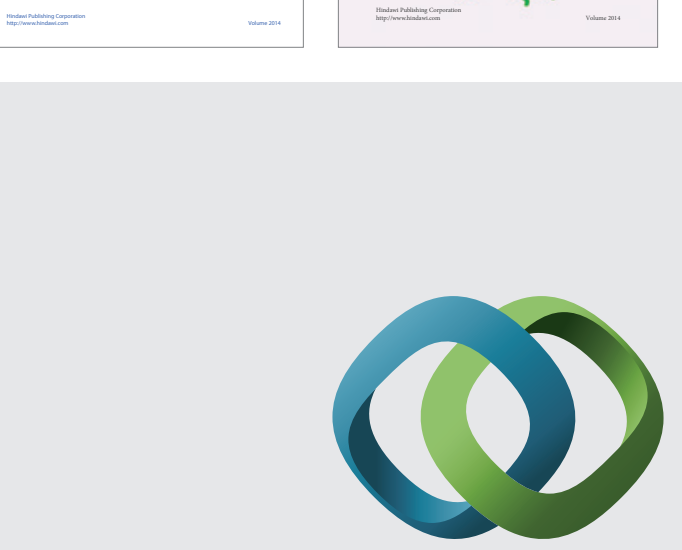

\section{Hindawi}

Submit your manuscripts at

http://www.hindawi.com
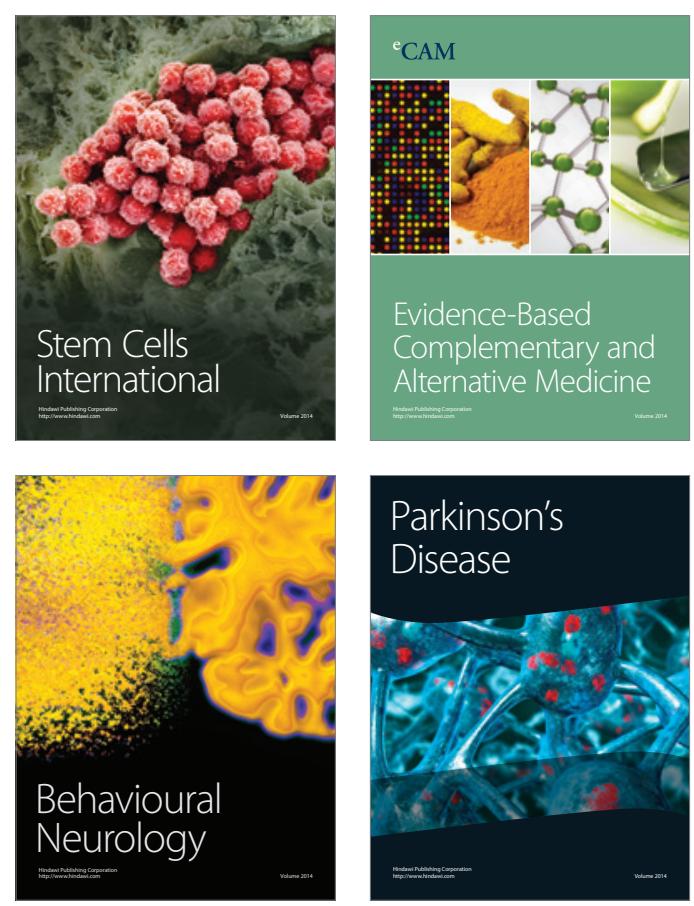

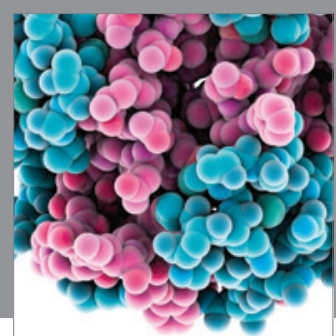

Journal of
Diabetes Research

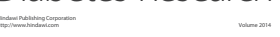

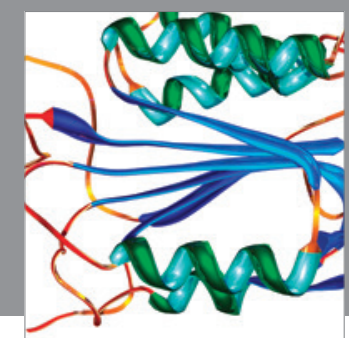

Disease Markers
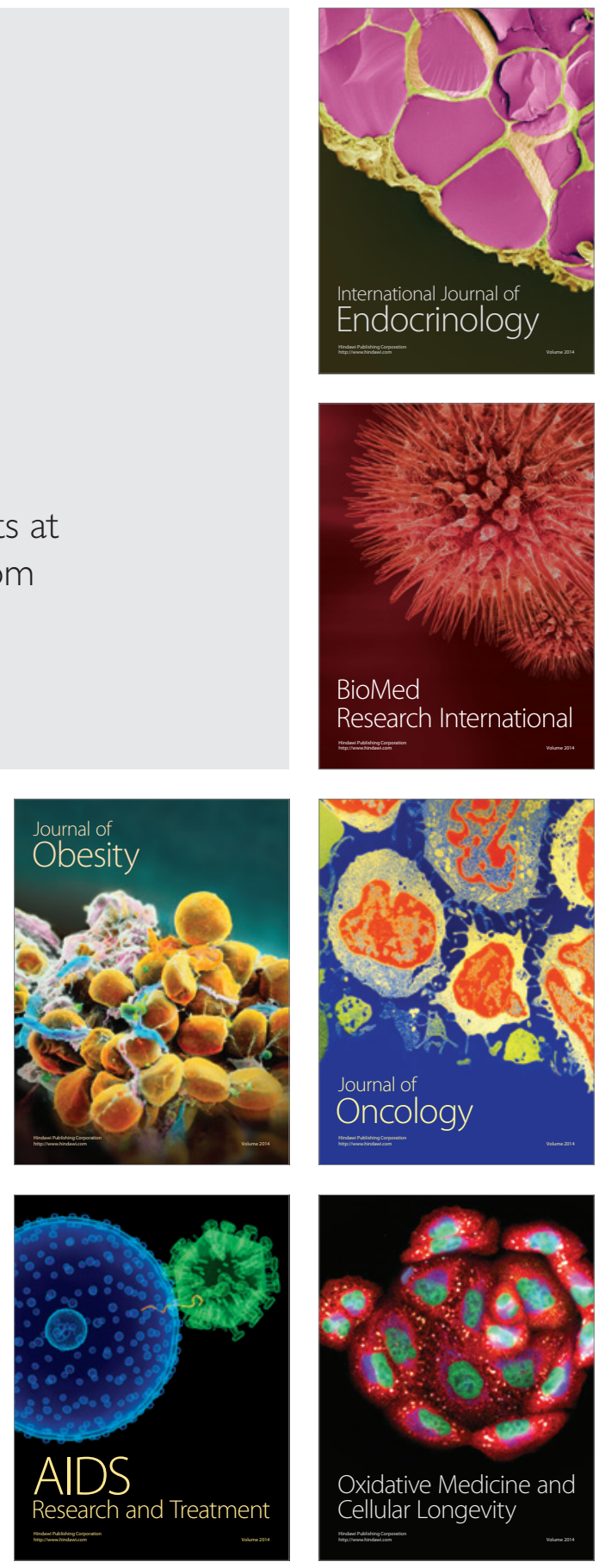